\title{
Electrical behavior of chemically grown lanthanum ferrite thin films
}

\author{
M.G.A. Ranieri ${ }^{\mathrm{a}}$, M. Cilense ${ }^{\mathrm{a}}$, E.C. Aguiar ${ }^{\mathrm{a}}$, C.C. Silva ${ }^{\mathrm{b}, 1}$, A.Z. Simões ${ }^{\mathrm{a}, \mathrm{b}, *, 1}$, E. Longo ${ }^{\mathrm{b}, 1}$ \\ ${ }^{a}$ Laboratório Interdisciplinar em Cerâmica (LIEC), Departamento de Físico-Química, Instituto de Química, UNESP, CEP 14800-900 Araraquara, SP, Brazil \\ ${ }^{\mathrm{b}}$ Universidade Estadual Paulista, Unesp, Faculdade de Engenharia de Guaratinguetá, Av. Dr. Ariberto Pereira da Cunha, 333, Bairro Pedregulho, \\ CEP 12516-410 Guaratinguetá, SP, Brazil
}

Received 23 July 2015; received in revised form 30 September 2015; accepted 4 October 2015

Available online 21 October 2015

\begin{abstract}
Perovskite structured oxides are important functional materials often used for the development of modern devices. To extend their applicability, these materials need to be scalably and efficiently grown in the form of thin films. In this work, perovskite structured thin films of nanograined $\mathrm{LaFeO}_{3}$ (LFO) were chemically grown using polymeric precursors on Pt substrates. The thin films were characterized by X-ray diffraction, field-emission scanning electron microscopy, atomic force microscopy, and transmission electron microscopy. The electrical properties of the films were also measured. The homogeneous LFO thin films synthesized at a sintering temperature of $500{ }^{\circ} \mathrm{C}$ in $2 \mathrm{~h}$ contained grains with lateral dimensions of about $68 \mathrm{~nm}$ and $356 \mathrm{~nm}$ in thickness. The dielectric permittivity and dielectric loss measurements of the sample indicated only a slight dispersion in the frequency because of the lower two-dimensional stress in the plane of the film. The nanograined LFO semiconductor thin films showed a room temperature magnetic coercive field, which rendered them magnetically soft. The electrical characterization of the films, including temperature-dependent conductivity and thermopower confirmed $p$-type conduction and the mobility activation energy was measured to be $0.96 \mathrm{eV}$. A strong magnetization with a remnant magnetization of $\sim 60 \mathrm{emu} / \mathrm{g}$ was observed in the LFO films, indicating the uncompensated spin magnets moments of the $\mathrm{Fe}^{3+}$ ions.
\end{abstract}

(c) 2015 Elsevier Ltd and Techna Group S.r.l. All rights reserved.

Keywords: A. Ceramics; B. Chemical syntheses; B. Thin films; C. X-ray diffraction

\section{Introduction}

Multiferroic (MF) materials, which exhibit simultaneous coexistence of magnetic (ferro/antiferromagnetic) and electric (ferro/ antiferro electric) orderings have gained widespread interest because the onset of magnetoelectric coupling in these materials occurs in the same phase. MFs have various potential applications in solid oxide fuel cells, non-volatile magnetic memory devices, and ultrasensitive magnetic read-heads of modern hard disk drives [1-3]. The coexistence of ferroelectricity and ferro/ antiferromagnetism and the unique coupling behavior renders MFs potential candidates for the design of new functional sensors and multistate devices [4]. High-quality nanocrystalline rare earth orthoferrites, especially $\mathrm{LnFeO}_{3}(\mathrm{Ln}=\mathrm{a}$ lanthanide element),

*Corresponding author.

E-mail address: alezipo@yahoo.com (A.Z. Simões).

${ }^{1}$ Tel.: +551231232765. have drawn much attention because of their unique physical and chemical properties for various applications [5]. Most ferroelectrics and multiferroic materials are oxide perovskites with a general stoichiometry $\mathrm{ABO}_{3}$. In perovskite structured materials, the small cations (B) are at the center of an octahedron of oxygen anions and the large cations (A) are at the unit cell corners. The high local internal electric field produces spontaneous polarization, leading to the onset of ferroelectric ordering. Further, the cation B-oxygen-cation-B angle is nearly $180^{\circ}$, which induces superexchange interaction, leading to the onset of magnetic ordering in the same system. Many compounds with the $\mathrm{ABO}_{3}$ stoichiometry crystallize with an orthorhombic distortion in the perovskite structure and $\mathrm{LaFeO}_{3}$ (LFO) is a prototype of this series. The crystal structures of these materials are derived from the standard cubic structure by the distortion of the $\mathrm{BO}_{6}$ octahedral. These perovskites are of particular interest because of their relatively simple crystal structure in combination with 
excellent magnetic, piezoelectric, optical, catalytic, and dielectric properties [6].

LFO is a semiconducting perovskite oxide [7,8], which has been extensively studied because of its potential applicability in gas sensors, electrodes of solid-state fuel cells, spin valves, and other exchange bias applications in magnetic memory, or for oxygen sensing $[4,7-15]$. LFO with antiferromagnetic characteristics $[9,10]$ crystallizes in an orthorhombically distorted perovskite structure with lattice parameters $a=5.557 \AA, b=5.5652 \AA$, and $c=$ $7.8542 \AA$ [14]. The Néel temperatures of thin film and bulk LFO are as high as 670 and $740 \mathrm{~K}$, respectively [10]. The antiferromagnetism and an extremely high ordering temperature $\left(T_{\mathrm{N}}\right)$ of LFO are very promising for its application in the storage industry, spin valves, exchange bias applications, and heterostructures of magnetic/magnetic and magnetic/electric films [11]. In this work, we have used a solution-based method widely used to prepare thin oxide films for the preparation of LFO thin films. Solution-based preparation methodologies involve either sol-gel processing or decomposition of organometallics. Various types of sol-gel processing include colloidal sol-gel processing, synthesizing inorganic polymeric gels from organometallic compounds, and formation of an organic polymeric complex which originates the polymeric precursor method (PPM). PPM can involve [16-18] in situ polymerization of organometallic monomers or the preparation of a viscous solution containing metal ions, polymers, and a solvent. This viscous solution can be easily converted into a thermoplastic gel at high polymer concentrations. In situ PPM has been used extensively to obtain ceramic powders with small particles and a single phase [19].

This method was originally developed by Pechini [20] and is based on the chelation of a metallic cation by a carboxylic acid (such as citric acid) and further polymerization, which is promoted by the addition of ethylene glycol, leading to polyesterification. To obtain crack-free films, process parameters, including solution viscosity and ionic concentration, substrate-film adhesion, heating conditions, preparation atmosphere, substrate, and withdrawal speed need optimization. Differences between the thermal expansion coefficients of the film and the substrate also influence PPM [21,22]. In this paper, we report, to the best of our knowledge, the first systematic study on the structural, morphological, and electrical characteristics of LFO thin films obtained by PPM. We have also investigated the magnetic behavior of orthorhombic LFO structures deposited on $\mathrm{Pt} / \mathrm{Ti} / \mathrm{SiO}_{2} / \mathrm{Si}$ substrates by PPM.

\section{Experimental procedure}

The LFO thin films were prepared by the PPM, as described elsewhere [23]. The precursor solutions of lanthanum and iron were prepared by adding and stirring the raw materials $\left(\mathrm{La}_{2} \mathrm{CO}_{3}\right.$-Merck $99.5 \%$ and $\mathrm{Fe}(\mathrm{NO})_{3} \cdot 9 \mathrm{H}_{2} 0$ - Merck $\left.99.9 \%\right)$ in to ethylene glycol and concentrated aqueous citric acid under heating. Appropriate quantities of the solutions of $\mathrm{La}$ and $\mathrm{Fe}$ were mixed and homogenized by stirring at $90{ }^{\circ} \mathrm{C}$. The molar ratio of metal:citric acid:ethylene glycol was 1:4:16. The viscosity of the resulting solution was set to $20 \mathrm{cP}$ by adjusting the water content using a Brookfield viscometer. The films were spin-coated from the deposition solution onto a Pt (111)/Ti/ $\mathrm{SiO}_{2} / \mathrm{Si}$ substrate. The LFO films completely crystallized after annealing for $2 \mathrm{~h}$ at $500{ }^{\circ} \mathrm{C}$. Multilayered films were obtained by spin-coating the deposition solution 10 times on the surface of the substrate at $5000 \mathrm{rpm}$. The films were annealed at $300{ }^{\circ} \mathrm{C}$ for $1 \mathrm{~h}$ at a heating rate of $3{ }^{\circ} \mathrm{C} / \mathrm{min}$ in a conventional furnace and later crystallized at $500{ }^{\circ} \mathrm{C}$ for $2 \mathrm{~h}$ in static air environment. The number of layers was set as a function of the desired thickness to achieve the desired electrical properties. The films were analyzed by X-ray diffraction (XRD) at room temperature (using a Rigaku-DMax 2000PC diffractometer) with $\mathrm{Cu}-\mathrm{K} \alpha$ radiation in the $2 \theta$ range $20-60^{\circ}$ at $0.3 \% \mathrm{~min}$. The crystallite size (d) of the films was calculated using the Scherrer equation $d=k \lambda / \beta \cos \theta$, where $k$ is a constant, $\lambda$ is the X-ray wavelength, and $\beta$ is the full width at half maximum (FWHM) of the maximum reflection measured from a slow scan, and $\theta$ is the diffraction angle of the main peak. The thickness of the annealed films was measured using scanning electron microscopy (SEM, Topcom SM-300) by imaging the transverse section using backscattered electrons. Three measurements were acquired to obtain the average thickness, which was $\sim 300 \mathrm{~nm}$. The microstructural characterization of the films was carried out using transmission electron microscopy (TEM, Philips CM-20). The surface morphology of the thin films was characterized by atomic force microscopy (AFM) in the tapping mode (Nanoscope IIIa-Bruker).

Further, X-ray photoelectron spectroscopy (XPS, PHI-5702 multifunction instrument) was carried out on the samples using an Al-K $\alpha$ X-ray source with a passing energy of $29.35 \mathrm{eV}$. The pressure in the chamber during the experiments was about $4.5 \times 10^{-9}$ Torr. The binding energy calibration scale was controlled using the O $1 s$ peak, which appears in the XPS profiles of the as-grown samples.

For electrical measurements, a top Au electrode $(0.5 \mathrm{~mm}$ in diameter) was sputtered through a shadow mask at room temperature. After the deposition of the top electrode, the film was annealed in a tube furnace under oxygen at $300{ }^{\circ} \mathrm{C}$ for $1 \mathrm{~h}$ to decrease the oxygen vacancies. The electrical properties of the capacitor $\left(\mathrm{Au} / \mathrm{LFO} / \mathrm{Pt} / \mathrm{Ti} / \mathrm{SiO}_{2} / \mathrm{Si}\right.$ ) were measured on a Radiant Technology RT6000 A tester equipped with a micrometer probe station in a virtual ground mode at $60 \mathrm{~Hz}$. The dielectric properties of the capacitor were measured with an HP4192A impedance/gain phase analyzer under zero bias at room temperature as a function of frequency in the range from $10 \mathrm{kHz}$ to $1 \mathrm{MHz}$. The magnetization measurements were carried out using a vibrating sample magnetometer (VSM, Quantum Design ${ }^{\mathrm{TM}}$ ). The electrical conductivity $(\rho)$ measurements were obtained in air using a PC-controlled fourprobe direct current (DC) equipment. The $\rho$ value was obtained in the temperature $(T)$ range room temperature. The thermopower was evaluated using K-thermocouples (which measured the applied temperature gradient) and platinum electrodes (which measured the resulting voltages).

\section{Results and discussion}

Fig. 1 shows the XRD pattern of the LFO thin films deposited on $\mathrm{Pt} / \mathrm{Ti} / \mathrm{SiO}_{2} / \mathrm{Si}$ (100) substrates. The polycrystalline film exhibited a 


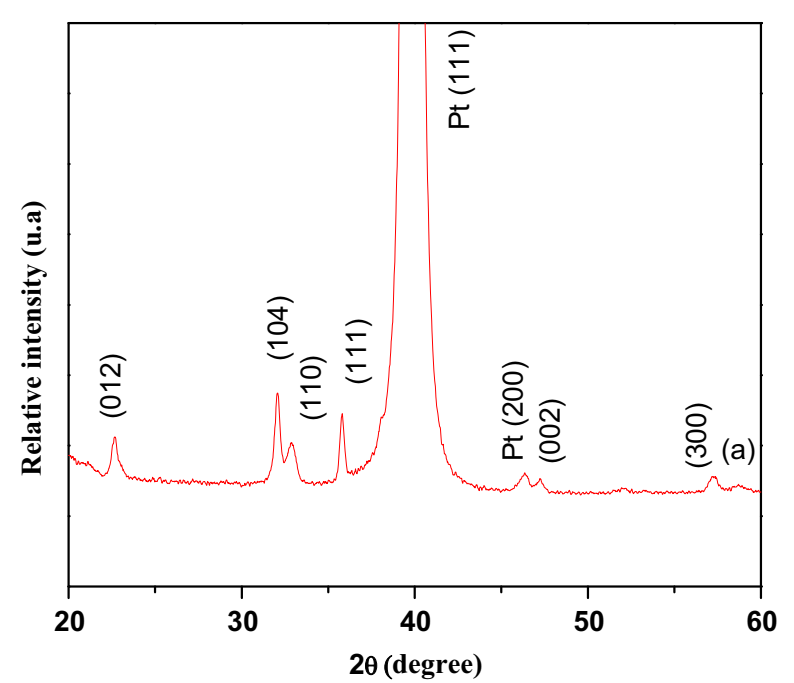

Fig. 1. X-ray diffraction patterns of the $\mathrm{LaFeO}_{3}$ thin films.

pure orthorhombic perovskite phase. These patterns were indexed to orthorhombic LFO (JCPDS file no. 74-2203) with lattice parameters (calculated from the XRD patterns) $a=0.554 \pm 0.004 \mathrm{~nm}$, $b=0.786 \pm 0.004 \mathrm{~nm}$, and $c=0.552 \pm 0.004 \mathrm{~nm}$. Furthermore, with the exception of the $\mathrm{Pt}$ (111) peaks, peaks corresponding to impurity phases, such as $\mathrm{FeO}$ and $\mathrm{Fe}_{2} \mathrm{O}_{3}$, were absent, indicating that single phase films were obtained by the chemical preparation used here. In addition, thermodynamically stable garnet $\left(\mathrm{Ln}_{3} \mathrm{Fe}_{5} \mathrm{O}_{12}\right)$ phases were absent in the XRD patterns, confirming the high purity of the products. This suggests that the annealing temperature can eliminate compositional fluctuations and the conversion of $\mathrm{Fe}^{3+}$ to $\mathrm{Fe}^{2+}$, which influence the appearance of secondary phases. The polycrystalline nature of the film can be attributed to differences in the nucleation energy between the antiferromagnetic material and the metallic electrode. The $d$ of the LFO film was calculated using the Debye-Scherrer equation as $37 \mathrm{~nm}$.

To confirm the thickness and surface morphology, surface and cross section SEM was carried out. Fig. 2 shows that the film was composed of uniform fine grains, several tens of $\mathrm{nm}$ in dimensions. Distinct grain boundaries were absent, which may be related with the low leakage current density because the opening of grain boundaries is suggested to lead to an increase of such parameter (Fig. 2(a)). Low annealing temperatures may also contribute to the low leakage current density because annealing at high temperatures can produce many defects, which can result in high leakage. Moreover, the surface was compact and smooth and Fig. 2(b) indicates the film thickness to be about $356 \mathrm{~nm}$.

The surface morphology of the LFO thin films was evaluated by AFM (Fig. 3), which indicated a homogeneous surface. Hence, the PPM allows for the preparation of LFO films with controlled morphology. The average surface roughness of the LFO film with an average grain size of $68 \mathrm{~nm}$ was $5.5 \mathrm{~nm}$. The PPM used in this study was found to be effective in improving the surface morphology of the LFO films because the precursor film underwent optimized nucleation and growth process, resulting in films with a homogeneous and dense microstructure.

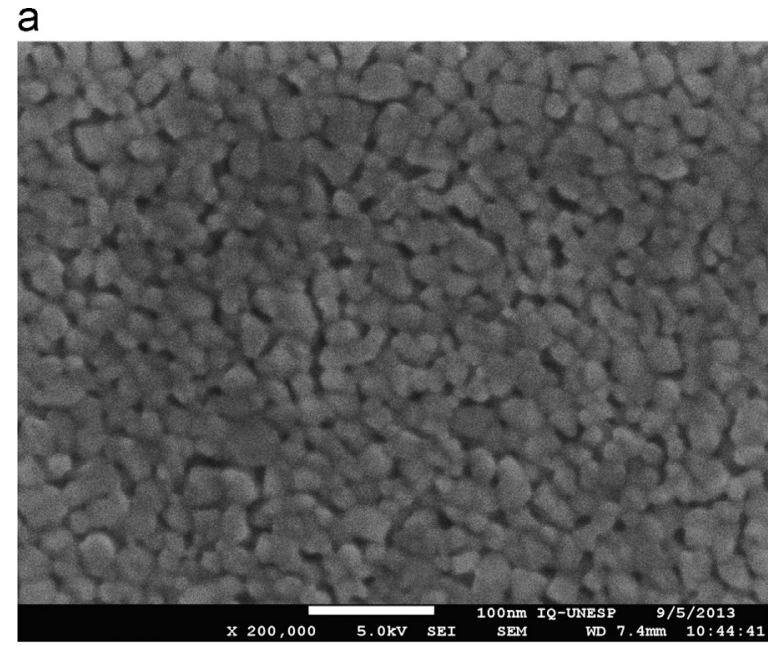

b

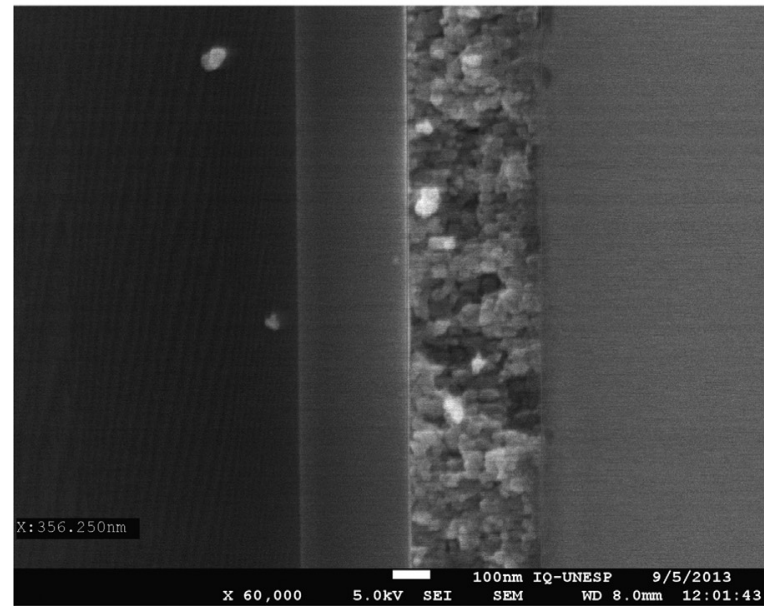

Fig. 2. (a) Surface and (b) cross section scanning electron microscopy images of the $\mathrm{LaFeO}_{3}$ thin films.

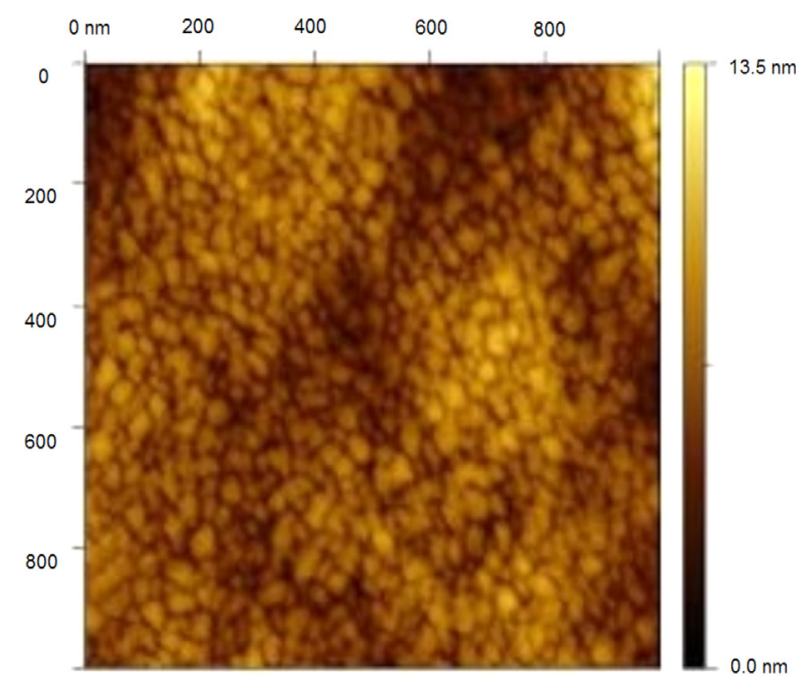

Fig. 3. Atomic force microscopy images of the $\mathrm{LaFeO}_{3}$ thin films.

The film morphology was uniform and the surface was smooth, indicating growth by a two-dimensional nucleation process. In addition, the homogeneous microstructure of such films allowed 
a

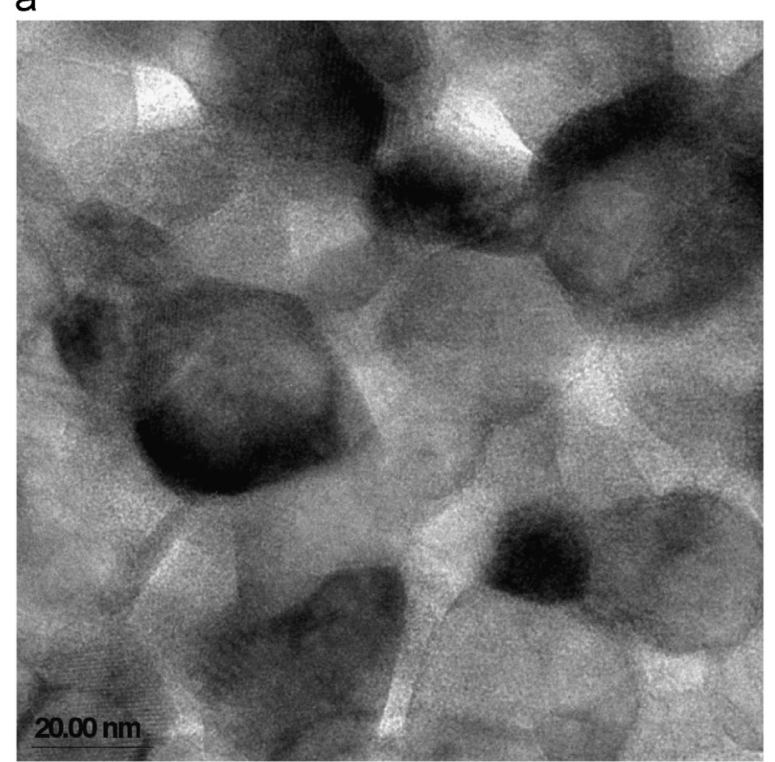

b

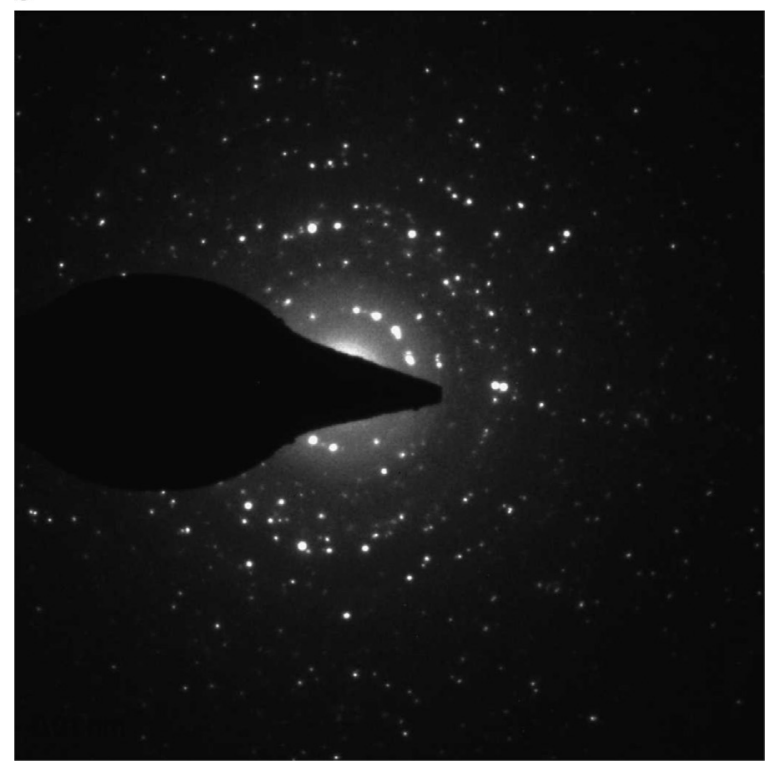

Fig. 4. (a) Transmission electron micrographs and (b) selected area electron diffraction patterns of the $\mathrm{LaFeO}_{3}$ thin films.

for the application of a uniform voltage, which significantly affected the measured electrical properties can be applied uniformly onto it.

The LFO film microstructure was evaluated by TEM and selected area electron diffraction (SAED) (Fig. 4(a) and (b)). TEM clearly demonstrated the polycrystallinity of the grains in the plane of the films. The grains were uniformly shaped and the grain size estimated from the dark-field images was about $70 \mathrm{~nm}$. Small pores (less than $10 \mathrm{~nm}$ in size) were present within the grains and along the grain boundaries. The small grains suggest suppression of oxygen vacancy concentration, which results in slower oxygen ion motion and consequently, lower grain growth rate. In addition, the small nucleation cores aggregated into larger grains, leading to a smoother surface (as shown in the AFM image). However, the grain boundaries were clearly distinguishable. Hence, the smaller grain size obtained can result from low crystallization temperatures because of the differences in the chemical bond strength between the $\mathrm{Fe}-\mathrm{O}$ and $\mathrm{La}-\mathrm{O}$ atoms. Further, the TEM analysis suggested the poor crystallinity of the material and the presence of a non-crystalline phase was evident. Further, the electron diffraction patterns indicated strong randomness in the atomic positions, as previously observed in the XRD results. Therefore, we assumed that the crystallization temperature exerts a strong effect on the crystal structure of the LFO films.

Generally, we assumed that conduction in LFO is related to electrons hopping from $\mathrm{Fe}^{2+}$ to $\mathrm{Fe}^{3+}$ with oxygen vacancies present in the lattice acting as a bridge between $\mathrm{Fe}^{2+}$ and $\mathrm{Fe}^{3+}$ (and hence, playing an important role in the electrical conduction) [24]. To identify the chemical bonding in LFO thin films, XPS was carried out. Fig. 5(a)-(c) shows the core-level XPS peaks of $\mathrm{La} 3 d, \mathrm{Fe} 2 p$, and $\mathrm{O} 1 s$ levels of the polycrystalline LFO films, respectively. The $3 / 2$ and $1 / 2$ spin-orbit doublet components of the $\mathrm{Fe} 2 p$ peaks were located at 711.1 and $724.6 \mathrm{eV}$, respectively. The XPS results show that LFO thin films consist of a single phase with $\mathrm{Fe}^{3+}$, which is consistent with the XRD results shown in Fig. 1. The 3/2 and 1/2 spinorbit doublet of the Fe $2 p$ peak located at 711.1 and $724.6 \mathrm{eV}$ were identified as $\mathrm{Fe}^{3+}$, respectively. In Fig. 5(b), the peaks of $\mathrm{Fe} 2 p 3 / 2$ level obtained at a binding energy of $710.9 \mathrm{eV}$ and a spin-orbital splitting at $13.5 \mathrm{eV}$ were consistent with other reports [25]. Peaks corresponding to $\mathrm{Fe}^{2+}$ and $\mathrm{Fe}$ were absent. The oxidation state of $\mathrm{Fe}$ was $3+$ exclusively, which was advantageous for producing LFO films with low leakage. The absence of $\mathrm{Fe}^{2+}$ suggested the reduction of oxygen vacancies in our films, which may be a reason for the enhanced ferroelectric properties observed, which will be discussed later. Fig. 4 (b) clearly shows the main peaks of $\mathrm{La} 3 d 3 / 2$ and $\mathrm{La} 3 d 5 / 2$ at binding energies of 834.6 and $851.4 \mathrm{eV}$, which are consistent with values published elsewhere $[25,26]$. The satellites at higher binding energies corresponded to the shake-up state of $\mathrm{La} 3 d$, resulting from a core hole with an electron transferred from the O $2 p$ valence band to an empty La $4 f$ orbit [27-29]. This indicated that the $\mathrm{La}$ and $\mathrm{Fe}$ ions in the LFO films were trivalent when the films were grown at $500{ }^{\circ} \mathrm{C}$.

The dielectric permittivity and the dissipation factor of the films are shown in Fig. 6(a). The dielectric measurements were carried out at room temperature as a function of frequency in the range from $10 \mathrm{kHz}$ to $1 \mathrm{MHz}$. The annealed films exhibited good dielectric properties with a relative dielectric permittivity of 290 and a dissipation factor $(\tan \delta$ ) of 0.06 at $1 \mathrm{MHz}$. The higher dielectric permittivity and a lower dissipation factor resulted from the increased density and closely packed uniform grains, as observed in Fig. 2(a). As can be seen from Fig. 6, the dielectric permittivity shows minimal dispersion with frequency, indicating low defect concentrations at the filmsubstrate interface. The low dispersion of the dielectric permittivity and the absence of any relaxation peak in $\tan \delta$ indicate an interfacial polarization of the Maxwell Wagner type and that the interfacial polarization arising from the electrode barrier is negligible. Fig. 6(b) shows the leakage current density as a function of the voltage measured at room 
a
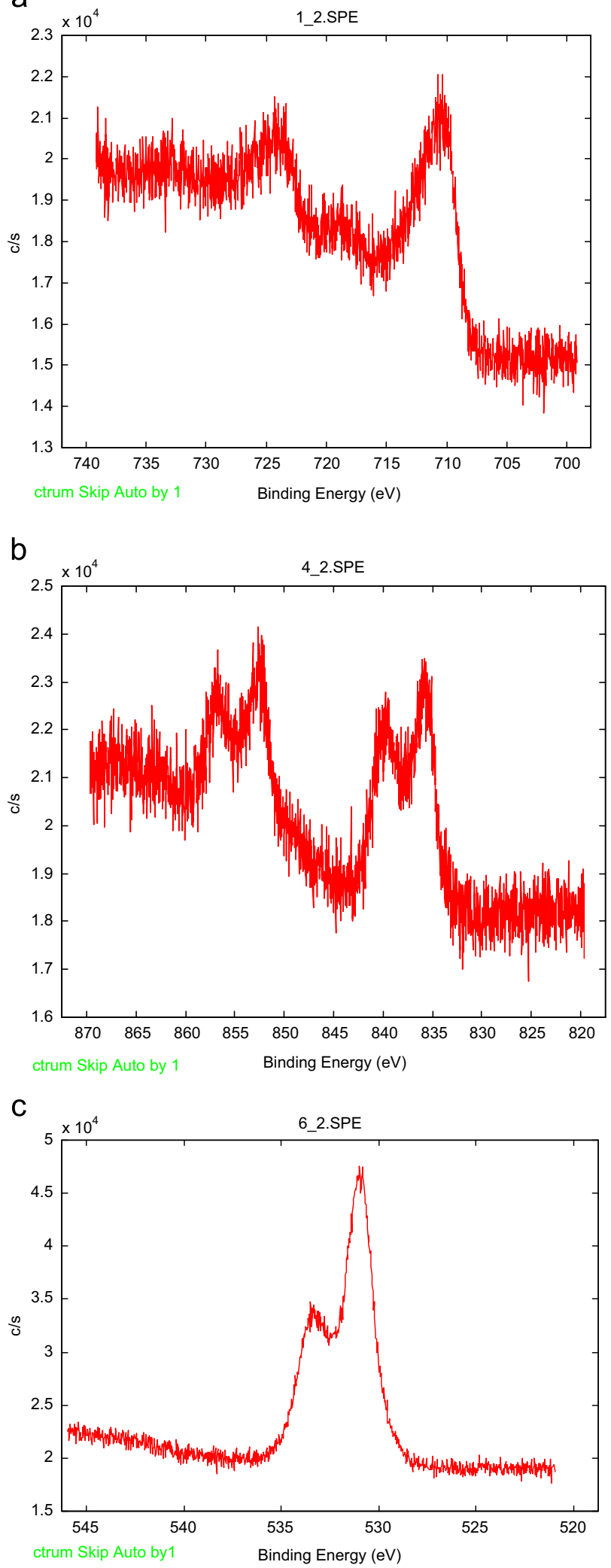

Fig. 5. The X-ray photoelectron core-level for $\mathrm{LaFeO}_{3}$ thin films. (a) $\mathrm{Fe} 2 p$ orbital, (b) La3d orbital and (c) O1s orbital.

temperature. The curve was recorded with a voltage step width of $0.1 \mathrm{~V}$ and elapsed time of $1.0 \mathrm{~s}$ for each voltage. The measured logarithmic current density $(\log J)$ versus the voltage
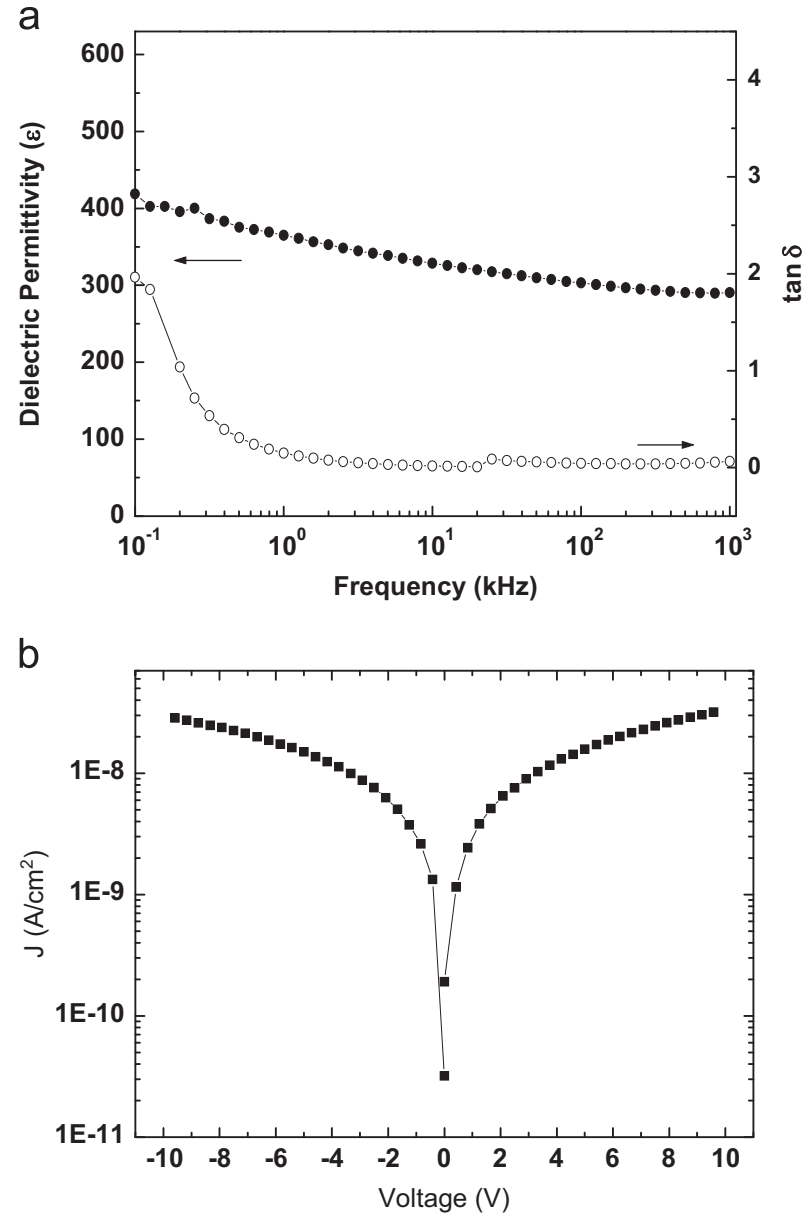

Fig. 6. (a) Dielectric permittivity and dielectric loss spectra of $\mathrm{LaFeO}_{3}$ thin films as a function of frequency and (b) leakage current density versus applied voltage.

$(V)$ is symmetric and shows two clearly different regions. The lower leakage current density of the LFO film may be attributed to the reduced number of electrons injected from the cathode at a rate faster than they can travel through the film. A low voltage was applied to overcome the larger repulsion forces caused by an increase in the amount of nonneutralized charges in the traps of the LFO film. This study demonstrates that the microstructure of the ferroelectric films affects their conductivity [30]. Since the conductivity is strongly affected by the characteristics of the film-electrode interface, the lower leakage current observed here may be probably attributed to differences in grain size, density, and lower stress in the plane of the film because of differences in the antiferromagnetic material and the interface. The current density increased linearly with the external voltage at low applied voltage, suggesting ohmic conduction. At higher field strengths, the current density increased exponentially, implying that at least a part of the conductivity originated from the Schottky or Poole-Frenkel mechanism. The leakage current density of the LFO films at $5.0 \mathrm{~V}$ was $10^{-10} \mathrm{~A} / \mathrm{cm}^{2}$.

The calculated value of the mobility activation energy of the LFO film is provided in Fig. 7. The LFO film exhibited a mobility activation energy of $0.96 \mathrm{eV}$, which was much higher than the value (of $0.20 \mathrm{eV}$ ) reported by Sarma et al. [31] for 


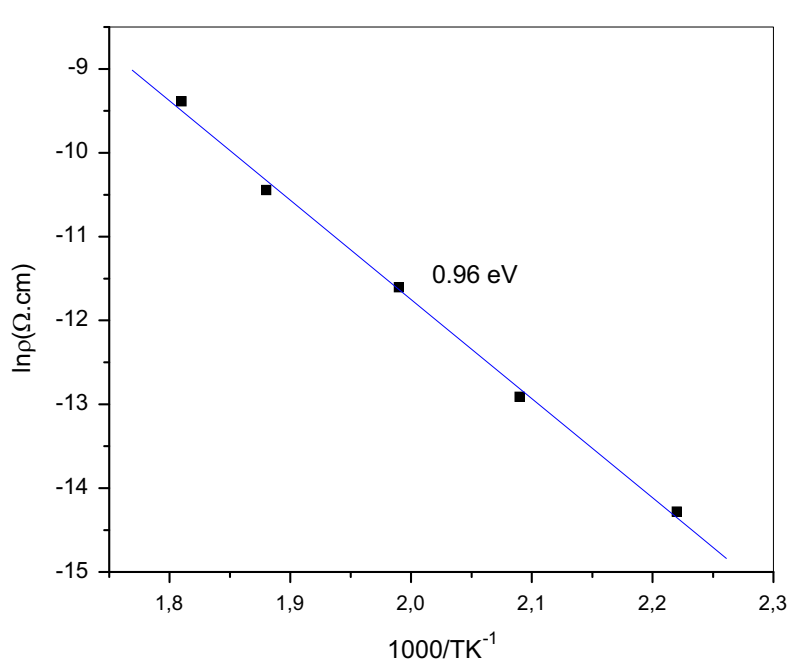

Fig. 7. Arrhenius curve for the $\mathrm{LaFeO}_{3}$ thin films.

nanostructured LFO thin films prepared by solid state reaction. The increase in $\rho$ with $T$ demonstrated the semiconducting nature of the LFO films, while the linearity between $\log (\rho)$ and $1000 / T$ indicated that the mobility was thermally activated, as expected for small polaron hopping. Therefore, the conduction occurred by thermally activated jumping of holes from $\mathrm{M}^{4+}$ to nearby $\mathrm{M}^{3+}$ via the intermediate oxide ions. It has also been reported [32] that the successive jump of the hole along the $\left(\mathrm{M}^{4+}-\mathrm{O}^{2-}-\mathrm{M}^{3+}\right)$ chain is slightly influenced by the Coulomb repulsion interaction because of the presence of $\mathrm{La}^{3+}$. These differences in electrical conductivity and mobility should be related to the differences in the band structure and energy gap. The highest hole concentration in LFO implies the lowest Fermi energy close to the valence band and consequently, the highest gap energy. This explanation was confirmed by calculating the band gap energy of the LFO film from the Arrhenius plot of fractional carrier concentration.

Magnetization $(M)$ versus field $(H)$ loops were recorded at $27{ }^{\circ} \mathrm{C}$ (Fig. 8). The magnetization of the film was observed with a remnant magnetization of $32.0039 \mathrm{emu} / \mathrm{g}$. A strong ferromagnetic response was measured and enhanced magnetization was observed in comparison to bulk specimens. Gehring [33] and Goodenough et al. [34] suggest that the statistical distribution of $\mathrm{Fe}^{3+}$ in the octahedra or creation of lattice defects might lead to bulk magnetization and weak ferromagnetism. The appearance of ferromagnetism in this compound may be attributed to either the canting of the antiferromagnetically ordered spins by a structural distortion [35-37] or the breakdown of the balance between the antiparallel sublattice magnetization of $\mathrm{Fe}^{3+}$ because of the substitution with metal ions of a different valency [38]. In this study, the magnetization of the LFO films non-linearly increased with the applied magnetic field, which is characteristic of antiferromagnets. This behavior ruled out the presence of any dominating paramagnetic particles in the sample. The non-linear curve is typical of ferri/ferromagnetic type of variation where the magnetization slowly approaches saturation. The maximum value of magnetization of the sample measured at an applied magnetic field of $13,800 \mathrm{G}$ was $60 \mathrm{emu} / \mathrm{g}$, while in the case of bulk LFO, the value of magnetization at the same magnetic field is $0.44 \mathrm{emu} /$

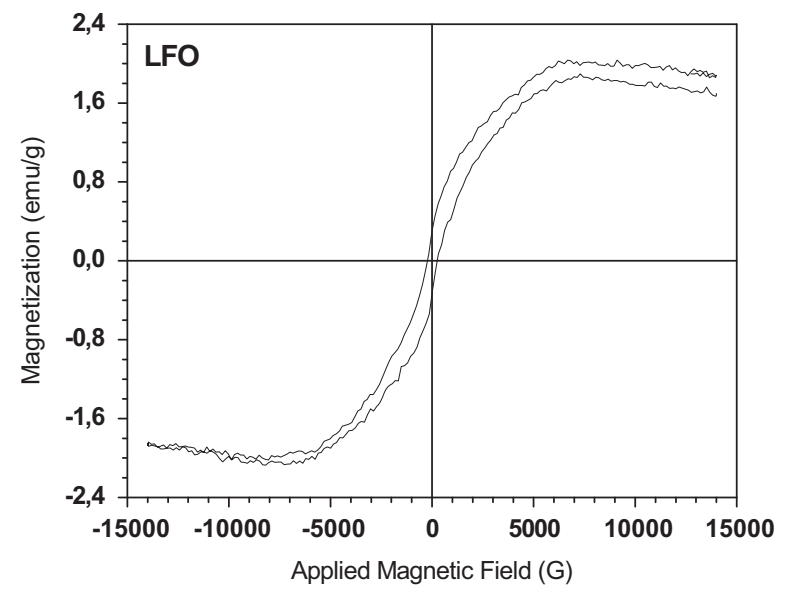

Fig. 8. Field dependencies of the magnetization obtained for the $\mathrm{LaFeO}_{3}$ thin films.

g [39]. Thus, the magnetization was enhanced by a factor of 136 , although the maximum field was only marginally high in our experiments. This observation may be attributed to the antiferromagnetic arrangement of the uncompensated spin magnetic moments of $\mathrm{Fe}^{3+}$ in the ferri/ferromagnetic system of LFO. The coercive magnetic field of the LFO film is $3799.66 \mathrm{G}$, which is indicative of its magnetically soft nature and demonstrates its suitability in device applications. In addition, LFO is a G-type antiferromagnet (AF) with $\mathrm{Fe}$ magnetic moments of $4.6 \mu \mathrm{B}$ and high Néel temperatures $\left(477^{\circ} \mathrm{C}\right)$. Hence, a similar magnetic order well above room temperature was observed in the LFO thin films synthesized here.

\section{Conclusions}

Pure perovskite LFO films were chemically synthesized by solution-based PPM. XRD and TEM showed that the films possess an orthorhombic structure with a dense and smooth surface. XPS showed that the $\mathrm{La}$ and $\mathrm{Fe}$ ions were trivalent when the films were grown at $500{ }^{\circ} \mathrm{C}$ in $2 \mathrm{~h}$. The high dielectric permittivity of the LFO films can be mainly attributed to the lower structural disorder and two-dimensional stress in the plane of the film. The lower leakage current measured at $5.0 \mathrm{~V}$ was $10^{-10} \mathrm{~A} / \mathrm{cm}^{2}$, which may be probably attributed to differences in grain size, density, and lower stress in the plane of the film because of the differences between the antiferromagnetic material and the interface. The LFO films exhibited $p$-type conduction (i.e., the holes were the dominant charge carriers). The magnetization of the LFO films at an applied magnetic field of $3799.66 \mathrm{G}$ was indicative of its magnetically soft nature and suitability for device applications.

\section{Acknowledgments}

This research project was financially supported by the Brazilian research funding agencies CNPq573636/2008-7, INCTMN2008/57872-1, and FAPESP2013/07296-2. 


\section{References}

[1] S.T. Shen, H.S. Weng, Comparative-study of catalytic reduction of nitricoxide with carbon-monoxide over the $\mathrm{La}_{1-x} \mathrm{Sr}_{x} \mathrm{BO}_{3}(\mathrm{~B}=\mathrm{Mn}, \mathrm{Fe}, \mathrm{Co}, \mathrm{Ni})$ catalysts, Ind. Eng. Chem. Res. 37 (7) (1998) 2654.

[2] V.V. Kharton, A.A. Yaremchenko, A.V. Kovalevsky, A.P. Viskup E.N. Naumovich, P.F. Kerko, Perovskite-type oxides for hightemperature oxygen separation membranes, J. Membr. Sci. 163 (2) (1999) 307.

[3] V.V. Kharton, A.A. Yaremchenko, E.N. Naumovich, Research on the electrochemistry of oxygen ion conductors in the former Soviet Union. II. Perovskite-related oxides, J. Solid State Electrochem. 3 (1999) 303.

[4] D. Kuscer, M. Hrovat, J. Holc, S. Bernik, D. Kolar, Some characteristics of $\mathrm{Al}_{2} \mathrm{O}_{3}$ - and $\mathrm{CaO}$ modified $\mathrm{LaFeO}_{3}$-based cathode materials for solid oxide fuel cells, J. Power Sources 61 (1996) 161.

[5] H. Xu, X. Hu, L. Zhang, Generalized low-temperature synthesis of nanocrystalline rare earth orthoferrites $\mathrm{LnFeO}_{3}(\mathrm{Ln}=\mathrm{La}, \mathrm{Pr}, \mathrm{Nd}, \mathrm{Sm}, \mathrm{Eu}$, Gd), Cryst. Growth Des. 8 (2008) 2061.

[6] S. Acharya, P.K. Chakrabarti, Some interesting observations on the magnetic and electric properties of $\mathrm{Al}^{3+}$ doped lanthanum orthoferrite $\left(\mathrm{La}_{0.5} \mathrm{Al}_{0.5} \mathrm{FeO}_{3}\right)$, Solid State Commun. 150 (2010) 1234.

[7] E. Traversa, S. Matsushuma, G. Okada, Y. Sadaoka, Y. Sakai, K. Watanabe, $\mathrm{NO}_{2}$ sensitive $\mathrm{LaFeO}_{3}$, thin films prepared by R.F. sputtering, Sens. Actuator B-Chem. 24-25 (1995) 661.

[8] N.N. Toan, S. Saukko, V. Lantto, Gas sensing with semiconducting perovskite oxide $\mathrm{LaFeO}$, Physica B 327 (2003) 279.

[9] R. Dogra, A.C. Junqueira, R.N. Saxena, A.W. Carbonari, J. MestnikFilho, M. Moralles, Hyperfine interaction measurements in $\mathrm{LaCrO}_{3}$ and $\mathrm{LaFeO}_{3}$ perovskites using perturbed angular correlation spectroscopy, Phys. Rev. B 63 (2001) 224104.

[10] A. Scholl, J. Stohr, J. Luning, J.W. Seo, J. Fompeyrine, H. Siegwart J.P. Locquet, F. Nolting, S. Anders, E.E. Fullerton, M.R. Scheinfein H.A. Padmore, Observation of antiferromagnetic domains in epitaxial thin films, Science 287 (2000) 1014.

[11] J. Nogues, I.K. Schuller, Exchange bias, J. Magn. Magn. Mater. 192 (1999) 203.

[12] Y. Yamada, T. Kusumori, H. Muto, Pinning effect of a $\mathrm{LaFeO}_{3}$ buffer layer on the magnetization of a $\mathrm{La}_{1-x} \mathrm{~Pb}_{x} \mathrm{MnO}_{3}$ layer, Appl. Phys. Lett. 80 (8) (2002) 1409

[13] D.S. Deng, X.F. Jin, Exchange bias in ferromagnetic/compensated antiferromagnetic bilayer, Phys. Rev. B 65 (2002) 172402. 65 (2002).

[14] S.E. Dann, D.B. Currie, M.T. Weller, M.F. Thomas, A.D. Al-Rawwas, The effect of oxygen stoichiometry on phase relations and structure in the system $\mathrm{La}_{1-x} \mathrm{Sr}_{x} \mathrm{FeO}_{3-\delta}(0 \leq x \leq 1,0 \leq \delta \leq 0.5)$, J. Solid State Chem. 109 (1994) 134.

[15] I. Hole, T. Tybell, J.K. Grepstad, I. Warnhus, T. Grande, K. Wiik, High temperature transport kinetics in heteroepitaxial $\mathrm{LaFeO}_{3}$ thin films, SolidState Electron. 47 (2003) 2279.

[16] N.G. Eror, H.U. Anderson, Polymeric precursor synthesis of ceramic materials, MRS Online Proc. Libr. 73 (1986) 571.

[17] S.G. Cho, P.F. Johnson, R.A. Condrate Sr., Thermal decomposition of (Sr,Ti) organic precursor during the Pechini process, J. Mater. Sci. 25 (1990) 4738.

[18] S. Kumar, G.L. Messing, Synthesis of barium titanate by a basic $\mathrm{pH}$ Pechini process, MRS Online Proc. Libr. 271 (1992) 95.

[19] M.P. Pechini, Method of Preparing Lead and Alkaline Earth Titanates and Niobates and Coating Method Using the Same to Form a Capacitor. U.S. Pat. No. 3330 697, July 11, 1967.

[20] M. Liu, D. Wang, Preparation of $\mathrm{La}_{1-z} \mathrm{Sr}_{z} \mathrm{Co}_{1-y} \mathrm{Fe}_{y} \mathrm{O}_{3-x}$ thin films, membranes, and coatings on dense and porous substrates, J. Mater. Res. 10 (1995) 3210.

[21] M. Okuyama, Y. Hamakawa, Preparation and basic properties of $\mathrm{PbTiO}_{3}$ ferroelectric thin films and their device applications, Ferroelectrics 63 (1985) 243.
[22] G. Biasotto, M.G.A. Ranieri, C.R. Foschini, A.Z. Simões, E. Longo J.A. Varela, M.A. Zaghete, Gas sensor applications of zinc oxide thin film grown by the polymeric precursor method, Ceram. Int. 40 (2014) 14991.

[23] A.Z. Simões, A.H.M. Gonzalez, M.A. Zaghete, J.A. Varela B.D. Stojanovic, Effects of annealing and viscosity on the surface roughness of PLZT thin films, Thin Solid Films 384 (2001) 132.

[24] W.M. Zhu, Z.-G. Ye, Effects of chemical modification on the electrical properties of $0.67 \mathrm{BiFeO}_{3}-0.33 \mathrm{PbTiO}_{3}$ ferroelectric ceramics, Ceram. Int. 30 (2004) 1435.

[25] J.F. Moulder, W.F. Stickle, P.E. Sobol, K.D. Bomben, J. Chastain, Handbook of X-ray Photoelectron Spectroscopy, Perkin-Elmer Corporation, Minnesota, 1992.

[26] X. Guo, Z. Chen, D. Cui, Y. Zhou, H.Z. Huang, H.X. Zhang, F. Liu, $\mathrm{K}$. Ibrahim, $\mathrm{H}$. Qian, The colossal magnetoresistance $\left(\mathrm{La}_{x} \mathrm{Sn}_{1-x}\right)_{y} \mathrm{MnO}_{3-\delta}$ films studied by $\mathrm{X}$-ray photoemission spectroscopy, Cryst. Growth 219 (2000) 404.

[27] A.J. Signorelli, R.G. Hayes, X-ray photoelectron spectroscopy of various core levels of lanthanide ious: the roles of monopole excitation and electrostatic coupling, Phys. Rev. B 8 (1973) 81.

[28] D.F. Mullica, C.K.C. Lok, H.O. Perkins, V. Young, X-ray photoelectron final-state screening in $\mathrm{La}(\mathrm{OH})_{3}$, a multiplet structural analysis, Phys. Rev. B 31 (1985) 4039.

[29] S.J. Oh, G.H. Kim, G.A. Sawatzky, H.T. Jonkman, Effect of holeinduced shakedown in the Auger spectrum of lanthanum, Phys. Rev. B 37 (1988) 6145.

[30] L.F. Goncalves, J.A. Cortés, M.G.A. Ranieri, F.B. Destro, M.A. Ramirez, A.Z. Simões, Fabrication and structural characterization of bismuth niobate thin films grown by chemical solution deposition, J. Mater. Sci.: Mater. Electron. 26 (2015) 1142.

[31] D.D. Sarma, N. Shanthi, S.R. Barman, N. Hamada, H. Sawada, K. Terakura, Band theory for ground-state properties and excitation spectra of perovskite $\mathrm{LaMO}_{3}(\mathrm{M}=\mathrm{Mn}, \mathrm{Fe}, \mathrm{Co}, \mathrm{Ni})$, Phys. Rev. Lett. 75 (1995) 1126.

[32] M.V. Patrakeev, J.A. Bahteeva, E.B. Mitberg, I.A. Leonidova V.L. Kozhevnikov, K.R. Poeppelmeier, Electron/hole and ion transport in $\mathrm{La}_{1-x} \mathrm{Sr}_{x} \mathrm{FeO}_{3-\delta}$, J. Solid State Chem. 172 (2003) 219.

[33] G.A. Gehring, On the microscopic theory of the magnetoelectric effect, Ferroelectrics 161 (1994) 275.

[34] J.B. Goodenough, J.M. Lango., Landolt-Börnstein Numerical Data and Functional Relationships in Science and Technology vol III/4a, Springer, New York, 1978.

[35] M.M. Kumar, S. Srinath, G.S. Kumar, S.V. Suryanarayana, Spontaneous magnetic moment in $\mathrm{BiFeO}_{3}-\mathrm{BaTiO}_{3}$ solid solutions at low temperatures, J. Magn. Magn. Mater. 188 (1998) 203.

[36] T. Kanai, S.I. Ohkoshi, A. Nakajima, T. Watanabe, K.A. Hashimoto, Ferroelectric ferromagnet composed of $(\mathrm{PLZT})_{x}\left(\mathrm{BiFeO}_{3}\right)_{1-x}$ solid solution, Adv. Mater. 13 (2001) 487.

[37] J. Wang, A. Scholl, H. Zheng, S.B. Ogale, L. Viehland, D.G. Schlom N.A. Spaldin, K.M. Rabe, M. Wuttig, L. Mohaddes, J. Neaton, U. Waghmare, T. Zhao, R. Ramesh, Response to comment on epitaxial $\mathrm{BiFeO}_{3}$ multiferroic thin film heterostructures, Science 307 (2005) 1203b.

[38] K. Ueda, H. Tabata, T. Kawai, Coexistence of ferroelectricity and ferromagnetism in $\mathrm{BiFeO}_{3}-\mathrm{BaTiO}_{3}$ thin films at room temperature, Appl. Phys. Lett. 75 (1999) 555.

[39] I. Wærnhus, P.E. Vullum, R. Holmestad, T. Grande, K. Wiik, Electronic properties of polycrystalline $\mathrm{LaFeO}_{3}$. Part I: experimental results and the qualitative role of Schottky defects, Solid State Ion. 176 (2005) 2783. 